\title{
Estudio piloto sobre la prevalencia de patología dual en pacientes en tratamiento en la Comunidad de Madrid
}

\section{Pilot study on the prevalence of dual pathology in community mental health and substance misuse services in Madrid}

\author{
Néstor Szerman Bolotner*; Francisco Arias \\ Horcajadas ${ }^{\star *}$; PABlo Vega Astudilloo**; Francisco \\ Babín Vich ${ }^{\star \star *}$; Beatriz Mesías Perez ${ }^{\star \star *}$; Ignacio Ba- \\ SURTE VILLAMOR ${ }^{\star * \star *}$; CONSUElo MORANT ${ }^{* * * * *}$; ENRIQUe-

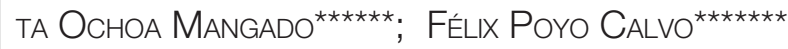 \\ Y GRUPO DE INVESTIGACIÓN DEL ESTUDIO PILOTO SOBRE \\ LA PREVALENCIA DE PATOLOGÍA DUAL EN LA COMUNIDAD DE \\ MADRID
}

\author{
* Unidad de Psiquiatría, Hospital Virgen de la Torre, Madrid. \\ ** Unidad de Psiquiatría Hospital Universitario Fundación Alcorcón. \\ Madrid. \\ *** Instituto Adicciones, Madrid-Salud. Madrid. \\ ${ }_{* \star \star \star}$ Unidad de Psiquiatría. Hospital Universitario Gregorio Marañón. \\ Madrid.

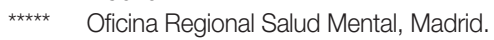

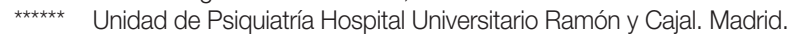

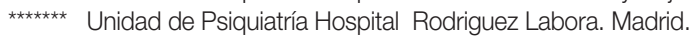

Enviar correspondencia a:

Francisco Arias Horcajadas. Unidad de Psiquiatría. Hospital Universitario Fundación Alcorcón. Avda. Budapest s/n. 28922

Alcorcón. Madrid. farias@fhalcorcon.es

\section{RESUMEN}

El objetivo ha sido valorar la presencia de diagnósticos comórbidos de trastornos mentales y adictivos de forma retrospectiva en la historia clínica de pacientes en tratamiento en las redes asistenciales de salud mental o de adicciones en la Comunidad de Madrid. Material y métodos: Se valoraron las historias clínicas de 400 pacientes en tratamiento en Centros de Atención al Drogodependiente (CAD), Centros de Atención Integral al Drogodependiente (CAID), Centros de Salud Mental (CSM) o servicios de psiquiatría de Hospitales de Madrid. Se recogieron de forma retrospectiva los datos de las últimas 20 historias clínicas de cada centro seleccionado. Resultados: La prevalencia de patología dual, considerando como tal la presencia de un diagnóstico actual de trastorno mental y de trastorno por uso de sustancias distinto al tabaco, fue del 34\%. Habia diferencias en la prevalencia entre las dos redes asistenciales, un 36.78\% de los pacientes en tratamiento en la red de drogas fueron considerados duales frente a un $28.78 \%$ en la red de salud mental. Había una asociación entre el diagnóstico de patología dual y el consumo perjudicial o dependencia de alcohol o cocaina pero no con el de heroina. Los trastornos mentales más frecuentes en los pacientes duales que en los no duales fueron los trastornos del humor, los trastornos de personalidad y la esquizofrenia. Conclusión: Por lo tanto, existe una elevada prevalencia de pacientes con patología dual entre los sujetos que buscan tratamiento, siendo mayor en la red de atención al drogodependiente y mayor entre aquellos con dependencia de alcohol o cocaína. Estos datos pueden ayudar a la hora de planificar los recursos asistenciales para este tipo de pacientes.

Palabras clave: patología dual, prevalencia, centros de salud mental, centros de atención a drogodependientes. recibido: enero 2011 aceptado: junio 2011

\section{ABSTRACT}

Aim: To evaluate retrospectively the comorbidity of mental and addictive disorders in community mental health and substance misuse services in Madrid. Methods: The medical records of 400 patients from mental health and substance misuse services in Madrid were evaluated. Records were examined for the last 20 patients from each service unit. Results: Dual pathology was constituted when a current diagnosis of mental and addictive disorders, excluding nicotine addiction, appeared on the patient's records. Prevalence of dual pathology was 34\%. There were differences in the prevalence figures for the two kinds of service: $36.78 \%$ in substance misuse services, and $28.78 \%$ in mental health services. There was an association of dual diagnosis with alcohol or cocaine dependence, but not with opioid dependence. The mental disorders more prevalent in dually diagnosed than in non-dually diagnosed patients were mood disorders, personality disorders, and schizophrenia. Conclusion: There is a high prevalence of dual pathology in those seeking treatment, being higher in substance misuse services than in mental health services, and higher in patients with alcohol or cocaine dependence. These findings could be of help in the planning of care resource policies for these patients.

Keywords: dual pathology, prevalence, mental health services, substance misuse services. 


\section{INTRODUCCIÓN}

L a patología dual hace referencia a la presentación en un mismo sujeto de un trastorno mental y un trastorno adictivo (Volkow, 2007). Diversos estudios epidemiológicos Ilevados a cabo principalmente en Estados Unidos indican que esta comorbilidad es muy elevada, siendo mayor en sujetos en tratamiento, y esta presencia concomitante de varios trastornos tiene implicaciones clínicas, evolutivas y terapéuticas relevantes (Compton, Thomas, Stinson y Grant, 2007; Kessler, Berglund, Demler, Jin, Merikangas y Walters, 2005; Kessler, Chiu, Demler, Merikangas y Walters, 2005; Regier, Farmer, Rae, Locke, Keith, Judd, et al., 1990; Swendsen, Conway, Degenhardt, Glantz, Jin, Merikangas, et al., 2010). A pesar de la elevada frecuencia, la relación entre ambos trastornos no está bien comprendida y el diagnóstico es complejo, en parte por el solapamiento de síntomas, por la dificultad en la distinción entre trastornos primarios e inducidos y por la ausencia de una exploración adecuada del trastorno comórbido una vez establecido un diagnóstico principal.

Dada la prevalencia estimada del problema y las consecuencias negativas para ambos trastornos por la presencia de dicha comorbilidad es necesario desarrollar medidas preventivas y terapéuticas adecuadas. Para ello, en primer lugar, necesitamos de información sobre la frecuencia del problema y la distribución de dicha comorbilidad en nuestro medio. Disponemos de escasos datos sobre la prevalencia de patología dual en población española en tratamiento y sobre la distribución de esta entre las dos redes asistenciales principales encargadas de la atención a estos pacientes, la red de asistencia al paciente adicto y la red de salud mental. Igualmente hay escasez de datos sobre los tipos de diagnósticos duales presentes en estos pacientes en nuestro medio. El conocimiento sobre la distribución de la patología dual y las características de estos sujetos puede ayudar a optimizar y organizar los recursos sanitarios.

El objetivo de este estudio fue valorar la presencia de diagnósticos comórbidos de trastornos mentales y adictivos de forma retrospectiva en la historia clínica de pacientes en tratamiento en la red asistencial de salud mental o de adicciones en la Comunidad de Madrid. Como objetivo secundario se analizaron las posibles relaciones entre trastornos mentales y adictivos. Estos datos forman parte de un estudio piloto que ayudó al diseño de un estudio transversal posterior en una muestra mayor. Este estudio piloto sirvió para valorar las variables de interés y para el cálculo del tamaño muestral del estudio posterior cuyos datos están recogidos y en proceso de análisis.

\section{MATERIAL Y MÉTODOS}

Se trata de un estudio descriptivo de los diagnósticos de trastornos mentales y adictivos a partir de datos de la historia clínica de 20 centros asistenciales de la Comunidad de Madrid (centros dependientes de la Agencia Antidroga de Madrid, del Instituto de Adicciones de Madrid Salud
- la Oficina Regional de Coordinación de Salud Mental). Se valoraron las historias clínicas de 400 pacientes en tratamiento en Centros de Atención al Drogodependiente (CAD), Centros de Atención Integral al Drogodependiente (CAID), Centros de Salud Mental (CSM) o servicios de psiquiatría de Hospitales de Madrid. Se incorporaron los datos de las últimas 20 historias clínicas de cada centro seleccionado desde una fecha establecida aleatoriamente igual para todos los centros. La fecha era previa al diseño del estudio. No hubo aleatorización de los centros participantes, se seleccionaron aquellos investigadores interesados en participar. Los centros de la red de drogodependencias atienden pacientes con diagnóstico principal o secundario de dependencia de drogas derivados desde atención primaria o especializada. Los CSM atienden pacientes con diagnóstico principal o secundario de trastorno mental distinto de la drogodependencia. Los pacientes con dependencia de alcohol como diagnóstico principal suele atenderse en los CSM, aunque si existe otra dependencia asociada pueden ser atendidos en los centros de la red de drogodependencias.

A partir de los datos de la historia clínica se recogieron de forma protocolizada variables sociodemográficas, historia de consumo de drogas y antecedentes de trastornos mentales, patologías médicas concomitantes, tratamientos recibidos para su trastorno mental o adictivo y diagnósticos actuales de los mismos recogidos en la historia clínica según criterios CIE-10. Se consideró que había un diagnóstico de patología dual cuando en la historia clínica estaba recogido un diagnóstico actual de trastorno mental y de trastorno por uso de sustancias distinto al tabaco.

Se realizó un análisis descriptivo de la muestra con el cálculo de medias y desviación estándar para las variables cuantitativas y frecuencias absolutas y relativas para las variables cualitativas. Se compararon variables cualitativas entre pacientes duales y no duales mediante la prueba de la chi-cuadrado o la prueba exacta de Fisher cuando no era aplicable la primera.

\section{RESULTADOS}

Las características sociodemográficas de la muestra y los diagnósticos actuales de trastorno mental y/o adictivo recogidos en la historia clínica se presentan en la tabla 1. La edad media del global de la muestra fue de 40 años (rango de 17 a 88 años), predominando los varones que eran un $68.75 \%$ de la muestra. Participaron 13 centros de la red de drogodependencias y 7 de la red de salud mental. La distribución de los pacientes por centros fue 261 (65.25\%) sujetos procedentes de centros de drogas y 139 (34.75\%) de la red de salud mental. Un centro de la red de salud mental aportó un paciente menos del previsto, mientras que un centro de la red de drogas aportó uno más del previsto que se utilizó para el análisis de los resultados.

Respecto a los diagnósticos actuales recogidos en la historia de consumo perjudicial o dependencia de drogas, 300 sujetos presentaban algún trastorno por uso de sustancias 
Tabla 1. Características de la muestra.

\begin{tabular}{|c|c|}
\hline & N (\%) o media (d.e.) [rango] \\
\hline Varones & $275(68.75)$ \\
\hline Edad (años) & 40.06 (d.e.=11.17) [17-88] \\
\hline \multicolumn{2}{|l|}{ Estado civil } \\
\hline Soltero & $196(49 \%)$ \\
\hline Casado & $115(28.75)$ \\
\hline Otros & $71(17.75)$ \\
\hline \multicolumn{2}{|l|}{ Estudios } \\
\hline Primarios & $120(30)$ \\
\hline Medios & $214(53.5)$ \\
\hline Superiores & $34(8.5)$ \\
\hline \multicolumn{2}{|l|}{ Situación laboral } \\
\hline Activo & $168(42)$ \\
\hline Paro & $112(28)$ \\
\hline Otros & $120(30)$ \\
\hline \multicolumn{2}{|l|}{ Procedencia } \\
\hline Red Salud Mental & $139(34.75)$ \\
\hline Red Drogas & $261(65.25)$ \\
\hline Patología médica* & $238(59.5)$ \\
\hline \multicolumn{2}{|c|}{$\begin{array}{l}\text { Características del consumo de sustancias } \\
\text { (diagnóstico actual recogido en la historia de consumo perjudicial o síndrome de dependencia) más frecuentes }\end{array}$} \\
\hline Algún diagnóstico actual de TUS** & $300(75)$ \\
\hline Alcohol & $153(38.25)$ \\
\hline Opioides & $119(29.75)$ \\
\hline Cannabis & $49(12.25)$ \\
\hline Sedantes & $25(6.25)$ \\
\hline Cocaína & $156(39)$ \\
\hline Otros estimulantes & $10(2.5)$ \\
\hline \multicolumn{2}{|c|}{ Características de los diagnósticos de trastornos mentales más frecuentes ( $\mathrm{CIE}-10$ ) } \\
\hline Algún diagnóstico actual recogido en la historia & $213(53.25)$ \\
\hline Esquizofrenia, Trastorno Esquizotípico o Trastorno de ideas delirantes & $24(6)$ \\
\hline Trastornos del humor & $108(27)$ \\
\hline Trastornos neuróticos, secundarios a situaciones estresantes y somatomorfos & $37(9.25)$ \\
\hline Trastornos de personalidad ${ }^{* *}$ & $56(14)$ \\
\hline
\end{tabular}

(TUS) (75\%), de los cuáles 238 (79.33\%) procedian de la red de drogas (CAD y CAID) y 62 (20.67\%) de la red de salud mental (CSM y servicios de psiquiatria de hospitales). Había 23 sujetos de la red de drogas donde no se recogía un diagnóstico actual de TUS, por no cumplir criterios para ello o por abstinencia de larga evolución. Los diagnósticos de TUS más prevalentes se reflejan en la tabla 1.

Entre los pacientes adictos un $90.33 \%$ presentaban policonsumo, es decir, consumo de más de una sustancia. Consi- derando la presencia de diagnóstico de TUS excluido tabaco, un 37.25\% presentaban más de un diagnóstico de TUS. Respecto a la dependencia de tabaco, esta se recogió en 76 sujetos (19\% del total).

Respecto a los diagnósticos actuales de trastornos mentales recogidos en la historia clínica, 213 sujetos (53.25\%) presentaban algún diagnóstico como se refleja en la tabla 1. De ellos 116 (54.46\%) procedian de la red de salud mental y 97 (45.54\%) de los dispositivos asistenciales de drogas. 
Igualmente habia 23 sujetos de la red de salud mental para los cuáles no figuraba un diagnóstico actual de trastorno mental, por ausencia de criterios para el mismo o por remisión del trastorno que motivó la asistencia.

La prevalencia de patología dual actual recogida en la historia clínica, es decir con un diagnóstico establecido de TUS excepto tabaco y un trastorno mental fue de 136 sujetos (34\% del total de la muestra). Estos se distribuian en 96 sujetos procedentes de la red de drogas (70.59\% de los pacientes duales y $36.78 \%$ de los pacientes atendidos en los centros de drogas) y 40 de la red de salud mental $(29.41 \%$ del total de duales y $28.78 \%$ de los pacientes atendidos en la red de salud mental).

Entre los pacientes duales los diagnósticos actuales de consumo perjudicial o dependencia de sustancias más fre- cuentes fueron: alcohol, cocaina y heroina por ese orden, como se refleja en la tabla 2. En los pacientes duales los diagnósticos de trastornos mentales actuales más frecuentes fueron: trastornos del humor; trastornos de personalidad y esquizofrenia (Tabla 2).

Al analizar la asociación entre los trastornos mentales y adictivos específicos más prevalentes se obtuvieron los siguientes resultados (Tabla 3): Había una relación estadísticamente significativa entre la presencia de trastornos del humor y consumo perjudicial o dependencia de alcohol al compararlo frente a los que no tenían trastornos del humor $(p<0.01)$, pero no se observó una asociación con el resto de sustancias. Entre los sujetos con diagnóstico de trastornos de personalidad, la asociación estadísticamente más relevante fue con el consumo perjudicial o dependencia de cocaína $(p<0.01)$.

Tabla 2. Diagnósticos de trastornos por uso de sustancias y mentales más prevalentes en pacientes con patología dual.

\begin{tabular}{lcc}
\hline & Pacientes duales $(\mathrm{n}=136) \mathrm{n}(\%)$ & No duales $(\mathrm{n}=264) \mathrm{n}(\%)$ \\
\hline Consumo perjudicial o dependencia de alcohol $^{*}$ & $78(57.3)$ & $75(29.07)$ \\
\hline Consumo perjudicial o dependencia de opioides $^{* *}$ & $46(33.8)$ & $73(27.65)$ \\
\hline Consumo perjudicial o dependencia de cocaína* & $72(52.94)$ & $84(32.56)$ \\
\hline Trastornos del humor $^{*}$ & $67(49.26)$ & $41(15.89)$ \\
\hline Trastornos de la personalidad* $^{*}$ & $46(33.82)$ & $10(3.88)$ \\
\hline Esquizofrenia $^{* *}$ & $13(9.56)$ & $11(4.26)$
\end{tabular}

${ }^{*} p<0.001 .{ }^{* *} p=0.2 .{ }^{* * *} p=0.04$.

Tabla 3. Relaciones entre los trastornos mentales y adictivos más prevalentes en los pacientes con patología dual*.

\begin{tabular}{|c|c|c|}
\hline & $\begin{array}{l}\text { Pacientes duales con trastornos } \\
\text { del humor }(n=67) n(\%)\end{array}$ & $\begin{array}{l}\text { Pacientes con patología dual sin } \\
\text { trastornos del humor }(n=69) n(\%)\end{array}$ \\
\hline Consumo perjudicial o dependencia de alcohol & $45(67.2)$ & $33(47.83)$ \\
\hline Consumo perjudicial o dependencia de opioides & $22(32.8)$ & 24 (34.78) \\
\hline \multirow[t]{2}{*}{ Consumo perjudicial o dependencia de cocaína } & $25(37.3)$ & $45(65.22)$ \\
\hline & $\begin{array}{l}\text { Pacientes duales con trastornos } \\
\text { de personalidad }(n=46) n(\%)\end{array}$ & $\begin{array}{l}\text { Pacientes con patología dual sin } \\
\text { trastornos de personalidad }(n=90) n(\%)\end{array}$ \\
\hline Consumo perjudicial o dependencia de alcohol & $26(56.5)$ & $52(57.8)$ \\
\hline Consumo perjudicial o dependencia de opioides & $20(43.5)$ & $26(28.9)$ \\
\hline \multirow[t]{2}{*}{ Consumo perjudicial o dependencia de cocaína } & $31(67.4)$ & $39(43.3)$ \\
\hline & $\begin{array}{l}\text { Pacientes duales con } \\
\text { esquizofrenia }(n=13) n(\%)\end{array}$ & $\begin{array}{l}\text { Pacientes con patología dual sin } \\
\text { esquizofrenia }(n=123) n(\%)\end{array}$ \\
\hline Consumo perjudicial o dependencia de alcohol & $5(38.5)$ & $73(59.3)$ \\
\hline Consumo perjudicial o dependencia de opioides & $4(30.8)$ & $42(34.1)$ \\
\hline Consumo perjudicial o dependencia de cocaína & $7(53.8)$ & $63(51.2)$ \\
\hline
\end{tabular}

*Todas las comparaciones mostraron diferencias no estadisticamente significativas, excepto la relación entre trastornos del humor y dependencia de alcohol y trastornos de personalidad y dependencia de cocaina $(p<0.01)$. 


\section{DISCUSIÓN}

En primer lugar, hay que señalar que las características de los pacientes duales cambian con el entorno clínico. Así hay más diagnósticos duales en la red de drogas que en la de salud mental, posiblemente por una mayor prevalencia de esta o puede que también por una mayor capacidad de detección de trastornos mentales entre los profesionales de adicciones que de trastornos adictivos entre los profesionales de salud mental. Igualmente puede influir la dificultad en diferenciar entre trastornos primarios e inducidos por drogas y es posible que en la red de drogas existan diagnósticos de trastornos inducidos como primarios. Si se aumentara la muestra procedente de salud mental es posible que las cifras de prevalencia se aproximaran, aunque consideramos que la diferencia es bastante destacada. Estas diferencias entre las dos redes se han objetivado previamente (Weaver, Madden, Charles, Stimson, Renton, Tyrer, et al, 2003), aunque se ha sugerido que los pacientes duales en la red de salud mental tienen una mayor gravedad psicopatológica (Primm, Gomez, Tzolova-Iontchev, Perry, Vu y Crum, 2000), variable esta que nosotros no hemos analizado. En este último trabajo, en una muestra más pequeña que la nuestra de pacientes en tratamiento en las dos redes asistenciales, observaron que había diferencias en el perfil psiquiátrico entre ambas redes y recomendaban que, para un manejo más efectivo, los programas de tratamiento integrado deberían ser conscientes de dichas diferencias y ajustar la provisión de servicios acorde a estas (Primm et al, 2000).

Consideramos que la cifra obtenida de prevalencia de patología dual es bastante destacada y subraya la importancia del problema, aunque debido a la recogida de datos a partir de la historia clínica y de forma retrospectiva, es previsible que la cifra esté infraestimada. De esta forma, se ha señalado que en servicios de salud mental hasta un $44 \%$ presentan TUS y en centros de atención a drogodependientes hasta un 75\% presentan trastornos mentales, destacando los autores un alto porcentaje de cuadros no detectados (Weaver et al, 2003). Con metodología menos rigurosa, en nuestro medio algunos autores han ofrecido cifras similares a las nuestras. En un estudio en unidades de agudos de psiquiatría señalaron una cifra similar a la nuestra de un 25\% de pacientes duales (Rodríguez-Jiménez, Aragüés, Jiménez-Arriero, Ponce, Muñoz, Bagney, et al., 2008). En una encuesta a profesionales que trabajaban con pacientes adictos, observaron que sobre un 34\% tenían comorbilidad psiquiátrica, siendo la depresión el cuadro psiquiátrico más prevalente $(21.6 \%)$ y la dependencia de opiáceos la menos asociada con la comorbilidad psiquiátrica (Gual, 2007).

Se podria suponer una sobrerrepresentación de la patología dual por la implicación de investigadores más interesados en la patología dual y, por tanto, más proclives a realizar un diagnóstico de este tipo, dado que no hubo selección aleatoria de los investigadores, sino que fue una participación voluntaria. De cualquier forma, la valoración se realizó de las historias del centro, no exclusivamente de las historias del investigador participante en el estudio.
Al valorar las relaciones específicas entre trastornos adictivos y mentales, este estudio ofrece algunos datos de interés, que hay que valorar con precaución dado que no era el objetivo principal del estudio. De esta forma, hemos observado una mayor presencia de patología dual entre los dependientes de alcohol y cocaína y menor con la dependencia de heroína. Esto coincide con los datos del estudio mencionado realizado en nuestro país (Gual, 2007). Es posible que los dependientes de heroína llevaran más tiempo en tratamiento y la psicopatología comórbida hubiera remitido, de cualquier forma estudios como el Epidemiologic Catchment Area (ECA) (Regier et al., 1990) ya señalaron que la dependencia de cocaína era la que presentaba una mayor asociación con los trastornos mentales.

También hemos observado una asociación entre los trastornos del humor y la dependencia de alcohol. De esta forma, en el estudio National Comorbidity Survey (NCS) (Kessler et al., 2005a, 2005b) la dependencia de alcohol se asoció fundamentalmente con los trastornos del humor y los de ansiedad. En el estudio National Epidemiologic Survey on Alcohol and Related Conditions (NESARC), los trastornos del humor se presentaban estrechamente asociados con los TUS en general (Grant, Stinson, Dawson, Chou, Dufour, Compton, et al., 2004). En otros trabajos se ha observado la asociación de la dependencia de alcohol con el trastorno bipolar o el trastorno depresivo mayor (Currie, Patten, Williams, Wang, Beck, El-Guebaly, et al, 2005; Merikangas, Herrell, Swendsen, Rössler, Ajdacic-Gross y Angst, 2008). Igualmente, otros autores señalaron que los trastornos del humor eran los más prevalentes en ambas estructuras asistenciales (Primm et al., 2000).

Por el contrario, la dependencia de cocaína se encontraba más asociada con el diagnóstico de trastornos de personalidad. Así, en el estudio ECA, la dependencia de cocaína es la que mayor asociación presentaba con patología mental y dentro de esta, la asociación más intensa era con los trastornos de personalidad (Regier et al., 1990). Igualmente, en el estudio NESARC los trastornos de personalidad presentaban una estrecha asociación con los TUS en general (Grant, Hasin, Stinson, Dawson, Chou, Ruan, et al., 2004).

En los pacientes con diagnóstico de esquizofrenia, se objetiva una alta prevalencia de TUS, superior al 50\%. En esta muestra la más frecuente es la dependencia de cocaína, después alcohol y, por último, heroína. No observamos una asociación específica con un TUS al compararlos con pacientes duales sin esquizofrenia debido a que el número de pacientes con esquizofrenia era muy bajo en el total de la muestra. De cualquier forma, se considera que sobre un $70-80 \%$ de los pacientes con esquizofrenia tienen un TUS comórbido (Westermeyer, 2006).

Al igual que otros estudios de prevalencia de la patología dual, hemos excluido la dependencia de tabaco para dicho análisis. Consideramos que la cifra de un 19\% de diagnósticos de dependencia de tabaco en nuestra muestra claramente representa un infradiagnóstico o una falta de recogida en la historia clínica de dicho diagnóstico si consideramos las cifras presentes en estos ámbitos (Grant et al., 2004b), lo 
que debería de motivar al clínico para mejorar el diagnóstico y la recogida de dicha información.

Como limitaciones del estudio podemos señalar la ausencia de una entrevista estructurada para el diagnóstico, se trata de una evaluación retrospectiva con el diagnóstico presente en la historia clínica. Esto posiblemente nos estaría infravalorando la presencia de patología dual. Esta limitación se ha pretendido corregir en el estudio epidemiológico posterior donde se ha incorporado una entrevista diagnóstica estructurada. No se han analizado otros trastornos mentales 0 adictivos menos prevalentes que pudieran haber sido de interés, el estudio se centró en tres TUS (alcohol, cocaína y heroína) y tres trastornos mentales (trastornos del humor, trastornos de personalidad y esquizofrenia).

De cualquier forma, hay que señalar el importante tamaño muestral a pesar de tratarse de un estudio piloto y la variedad de centros implicados, así desconocemos de otros estudios similares llevados a cabo en nuestro país, por lo que esta información sería pionera en nuestro medio.

El hecho de que la patología dual sea tan frecuente, fundamentalmente en la red de drogas, sugiere que es necesaria la adecuada formación de los profesionales de estos centros en el diagnóstico y tratamiento de los trastornos mentales o una comunicación adecuada entre las dos redes. Por otro lado, es importante concienciar a los profesionales de la red de salud mental en la importancia de la adecuada detección y manejo de los trastornos adictivos.

\section{AGRADECIMIENTOS}

El trabajo ha sido coordinado por la Sociedad Española de Patología Dual y financiado por el instituto de Adicciones de Madrid Salud, la Agencia Antidroga de la Comunidad de Madrid, la Oficina Regional de Salud Mental de Madrid y la Obra Social de Caja Madrid.

Grupo de Investigación del Estudio Piloto sobre la prevalencia de patología dual en la Comunidad de Madrid: Grupo del Instituto de Adicciones de Madrid: Purificación Camarena Rodríguez; Luis Fernando Cordero de Ciria; Marina Delgado García; Oscar David Galera García; Ignacio García Barquero; Nieves Lahuerta Galán; Rocio Molina Prado; Gerardo Pacheco Otoya; José Javier Rosa Mestre; Zita Carmen Sierra de Grado; Carmen Tosío González; Pablo Vega Astudillo.

Grupo de la Agencia Antidroga de la Comunidad de Madrid: Ana Bohigas Hurtado; Ma Victoria Lázaro Muñoz; Gregorio Martín Carmona; Susana Martínez-Osorio del Rio; José Morante del Peral; Celia del Pino Lidón.

Grupo del Servicio Regional de Salud Mental de la Comunidad de Madrid: Francisco Arias Horcajadas; Ignacio Basurte Villamor; Pedro Cuadrado Callejo; Martín Miguel Iribarren; Enriqueta Ochoa Mangado; Félix Poyo Calvo; Néstor Szerman Bolotner.

\section{REFERENCIAS}

Compton, W.M., Thomas, Y.F., Stinson, F.S. y Grant, B.F. (2007). Prevalence, correlates, disability, and comorbidity of DSMIV drug abuse and dependence in the United States: results from the national epidemiologic survey on alcohol and related conditions. Archives of General Psychiatry, 64, 566-576.

Currie, S.R., Patten, S.B., Williams, J.V., Wang, J., Beck, C.A., El-Guebaly, N. y Maxwell, C. (2005). Comorbidity of major depression with substance use disorders. Canadian Journal of Psychiatry, 50, 660-666.

Grant, B.F., Hasin, D.S., Stinson, F.S., Dawson, D.A., Chou, S.P., Ruan, W.J. y Pickering, R.P. (2004a). Prevalence, correlates, and disability of personality disorders in the United States: results from the national epidemiologic survey on alcohol and related conditions. Journal of Clinical Psychiatry, 65, 948-958.

Grant, B.F., Stinson, F.S., Dawson, D.A., Chou, S.P., Dufour, M.C., Compton, W., ... Kaplan, K. (2004b). Prevalence and co-occurrence of substance use disorders and independent mood and anxiety disorders: results from the National Epidemiologic Survey on Alcohol and Related Conditions. Archives of General Psychiatry, 61, 807-816.

Gual, A. (2007). Dual Diagnosis in Spain. Drug and Alcohol Review, $26,65-71$

Kessler, R.C., Berglund, P., Demler, O., Jin, R., Merikangas, K.R. y Walters, E.E. (2005a). Lifetime prevalence and age-of-onset distributions of DSM-IV disorders in the National Comorbidity Survey Replication. Archives of General Psychiatry, 62, 593-602.

Kessler, R.C., Chiu, W.T., Demler, O., Merikangas, K.R. y Walters, E.E. (2005b). Prevalence, severity, and comorbidity of 12-month DSM-IV disorders in the National Comorbidity Survey Replication. Archives of General Psychiatry, 62, 617-627.

Merikangas, K.R., Herrell, R., Swendsen, J., Rössler, W., Ajdacic-Gross, V. y Angst, J. (2008). Specificity of bipolar spectrum conditions in the comorbidity of mood and substance use disorders: results from the Zurich cohort study. Archives of General Psychiatry, 65, 47-52.

Primm, A.B., Gomez, M.B., Tzolova-lontchev, I., Perry, W., Vu, H.T. y Crum, R.M. (2000). Mental health versus substance abuse treatment programs for dually diagnosed patients. Journal of Substance Abuse Treatment, 19, 285-290.

Regier, D.A., Farmer, M.E., Rae, D.S., Locke, B.Z., Keith, S.J., Judd, L.L. y Goodwin, F.K. (1990). Comorbidity of mental disorders with alcohol and other drug abuse. Results from the Epidemiologic Catchment Area (ECA) Study. JAMA, 264, 2511-2518.

Rodríguez-Jiménez, R., Aragüés, M., Jiménez-Arriero, M.A., Ponce, G., Muñoz, A., Bagney, A., ... Palomo, T. (2008). Trastornos duales en pacientes psiquiátricos ingresados: prevalencia y caracteristicas generales. Investigación Clínica, 49, 195-205.

Swendsen, J., Conway, K.P., Degenhardt, L., Glantz, M., Jin, R., Merikangas, K.R., ... Kessler, R.C. (2010). Mental disorders as risk factors for substance use, abuse and dependence: results from the 10-year follow-up of the National Comorbidity Survey. Addiction, 105, 1117-28. 
Volkow, N.D (2007). Addiction and Co-ocurring mental disorders. Director's perspective. NIDA Notes, 21, 2.

Weaver, T., Madden, P., Charles, V., Stimson, G., Renton, A., Tyrer, P., ... Ford, C.(2003). Comorbidity of Substance Misuse and Mental Illness Collaborative study team. Comorbidity of substance misuse and mental illness in community mental health and substance misuse services. British Journal of Psychiatry, 183, 304-313.

Westermeyer, J. (2006). Comorbid schizophrenia and substance abuse: a review of epidemiology and course. American Journal of Addictions, 15, 345-355. 
